\title{
Estudos de Validação do Interpersonal Needs Questionnaire (INQ-15) para a População Portuguesa
}

\author{
Validation Studies of the Interpersonal Needs Questionnaire (INQ-15) to the \\ Portuguese Population
}

\author{
Sara Costa $^{1}$, Rui C. Campos $^{2}$, Ana Simões ${ }^{1}$ e Ana Sofia Pio ${ }^{1}$
}

\begin{abstract}
Resumo
O objetivo deste trabalho é apresentar estudos de validação do Interpersonal Needs Questionnaire (INQ-15) para a população portuguesa. Este instrumento operacionaliza os constructos perceção de ser um fardo e sentimentos de não pertença de acordo com a Teoria Psicológica Interpessoal do Suicídio de Joiner (2005). No primeiro estudo, a Análise em Componentes Principais demonstrou a existência de três fatores e verificou-se igualmente uma adequada consistência interna, validade convergente e validade referenciada pelo critério risco suicidário. O segundo estudo permitiu demonstrar a validade preditiva das escalas do INQ15 a cinco meses e a validade referenciada pelo critério ideação suicida. No terceiro, os resultados apoiam a validade discriminante das escalas do INQ-15 e confirmam a estrutura fatorial de três fatores, obtida no primeiro estudo. No último estudo, foi confirmada a estabilidade temporal das duas escalas do INQ-15 com 2 semanas de intervalo.
\end{abstract}

Palavras-chave: Interpersonal Needs Questionnaire 15, perceção de ser um fardo, sentimentos de não pertença, estudos de validação

\begin{abstract}
The aim of this research is to validate the Interpersonal Needs Questionnaire (INQ-15) for the Portuguese population. This measure assesses perceived burdensomeness and thwarted belongingness according to Joiner's (2005) Interpersonal-Psychological Theory of Suicide. In the first study, Principal Component Analysis demonstrated the existence of three factors and was also demonstrated an adequate internal consistency, convergent validity and criterion related to validity, using suicide risk as criteria. The second study demonstrated the predictive validity of the INQ-15 scales at five months and the criterion related to validity using suicide ideation as criteria. In the third study, results support the discriminant validity of the INQ-15 scales and confirm the factorial structure of three factors, obtained in the first study. In the last study, the temporal stability of the two INQ-15 scales within a 2 weeks interval was confirmed.
\end{abstract}

Keywords: Interpersonal Needs Questionnaire 15, perceived burdensomeness, thwarted belongingness, validation studies

Este estudo foi parcialmente financiado por fundos nacionais através da FCT - Fundação para a Ciência e a Tecnologia, I.P., no âmbito do projeto UID/CED/04312/2019.

\footnotetext{
${ }^{1}$ Departamento de Psicologia, Escola de Ciências Sociais, Universidade de Évora, Portugal

2 Departamento de Psicologia, Escola de Ciências Sociais e Centro de Investigação em Educação e Psicologia (CIEP-UE), Universidade de Évora, Portugal.Apartado 94, 7002-554 Évora. E-mail: rcampos@ uevora.pt Revista Iberoamericana de Diagnóstico y Evaluación - e Avaliação Psicológica. RIDEP · N53 · Vol.4 · 63-77 · 2019 ISSN: 1135-3848 print /2183-6051online
} 


\section{Introdução}

A Teoria Psicológica Interpessoal do Suicídio (Joiner, 2005) propõe uma explicação para o desejo de suicídio. Segundo Joiner (2005), o desejo suicidário resulta da presença simultânea de duas variáveis: os sentimentos de não pertença (thwarted belongingness) e a perceção de ser um fardo (perceived burdensomeness). Ainda assim, estas duas variáveis não explicam, porque é que alguns indivíduos podem passar ao ato, ou seja, porque é que alguém tenta suicidar-se ou morre por suicídio (Chu et al., 2017).

Os sentimentos de não pertença podem ser definidos como a ausência de uma experiência de envolvimento pessoal num sistema ou ambiente, de modo a que exista uma integração nesse contexto (Hagerty, Lynch-Sauer, Patusky, Bouwsema, \& Collier, 1992; Marty, Segal, Coolidge, \& Klebe, 2012). Importa salientar que os sentimentos de não pertença não são sinónimo de escassez ou ausência de interações e que a necessidade de pertença só é satisfeita quando as interações são frequentes e positivas (Van Orden et al., 2010). Por outro lado, a perceção de ser um fardo consiste numa perceção do indivíduo que tem subjacente a ausência de um sentimento de eficácia ou competência (Joiner, 2005). Esta dimensão psicológica diz respeito à perceção de que a existência do próprio acarreta encargos estáveis e permanentes para os familiares, amigos e/ou sociedade, sendo a morte vista como a única solução para o problema (Joiner, 2005; Joiner et al., 2005, 2009).

O Interpersonal Needs Questionnaire (INQ) foi desenvolvido para operacionalizar os dois construtos interpessoais da teoria de Joiner (2005). O INQ surge com o objetivo de permitir uma avaliação prévia à intervenção em crise, permitindo direciona-la para dois importantes fatores de risco: os sentimentos de não pertença e perceção de ser um fardo (Van Orden, Cukrowicz, Witte, \& Joiner, 2012). Originalmente, o INQ era composto por 25 itens, mas posteriormente, foram desenvolvidas cinco diferentes versões, que têm vindo a ser utilizadas na investigação [versão com 25 itens (Van Orden, 2009), versão com 18 itens (Marty, Segal, Coolidge, \& Klebe 2012), com 15 itens (Van Orden, Cukrowicz, Witte, \& Joiner 2012), com 12 itens (Freedenthal, Lamis, Osman,
Kahlo, \& Gutierrez, 2011) e com 10 itens (Bryan, 2011)]. O desenvolvimento de outras versões prendeu-se com a elevada multicolinearidade de alguns dos itens das duas escalas da versão de 25 itens (Van Orden et al., 2012). A versão que será o foco da presente investigação é a de 15 itens, já que é considerada a versão que proporciona resultados mais precisos dos dois construtos que pretende avaliar (Hill et al., 2015; Van Orden et al., 2012).

Diversos estudos empíricos de validação da forma de 15 itens do INQ, foram realizados: estudos relativos à estrutura fatorial, à precisão e validade e, estudos que relacionaram os resultados nas duas escalas da prova com variáveis psicopatologias e com a suicidalidade. Globalmente, estes trabalhos evidenciaram que a prova apresenta uma estrutura de dois fatores, uma consistência interna adequada, uma elevada estabilidade temporal em intervalos curtos de tempo e uma relação entre os construtos avaliados e variáveis psicopatologias, nomeadamente e, muito em particular, a suicidalidade (cf. Chu et al., 2017).

O presente trabalho tem como objetivo apresentar quatro estudos de validação da versão de 15 itens do Interpersonal Needs Questionnaire (INQ-15) para a população portuguesa. No primeiro estudo, com uma amostra de adultos da comunidade, será testada a estrutura fatorial da versão portuguesa através de Análise em Componentes Principais. Espera-se, tendo por base a Teoria Psicológica Interpessoal do Suicídio, operacionalizada no INQ-15, que se obtenham dois fatores relativos à perceção de ser um fardo e aos sentimentos de não pertença. Será também avaliada a consistência interna, a validade convergente e a validade referenciada pelo critério risco de suicídio das duas escalas do INQ-15. Espera-se que os sentimentos de não pertença e a perceção de ser um fardo se relacionem positiva e significativamente com os sintomas depressivos (e.g., Anestis et al., 2016; Baams, Grossman, \& Russel, 2015; Bell et al., 2018; Cole et al., 2013; Guidry \& Cukrowicz, 2016), com a dor psicológica (e.g., Campos \& Holden, 2015) e com risco suicidário (e.g., Anestis \& Joiner, 2011; Anestis, Bagge, Tull, \& Joiner, 2011; Anestis, Khazem, Mohn, \& Green, 2015; Joiner et al., 2009). Para estudar a validade referenciada pelo 
critério risco suicidário irá comparar-se os resultados nas duas escalas do INQ-15, dos indivíduos que obtiveram um resultado igual ou superior ao ponto de corte numa medida de risco suicidário, com os que obtiveram um resultado inferior. Espera-se que os indivíduos com resultados iguais ou superiores ao ponto de corte obtenham resultados significativamente mais elevados nas duas escalas do INQ-15.

No segundo estudo, realizado com uma amostra de estudantes universitários, será testada uma vez mais a consistência interna das duas escalas do INQ-15, a validade referenciada pelo critério ideação suicida e a validade preditiva. Espera-se que os indivíduos com resultados iguais ao ponto de corte numa medida de ideação suicida apresentem resultados significativamente mais elevados nas duas escalas do INQ-15. Espera-se igualmente, que num modelo longitudinal de path analysis, se verifique uma relação direta entre a perceção de ser um fardo e variações na ideação suicida ao longo de cinco meses e, uma relação indireta entre os sentimentos de não pertença e variações na ideação através da perceção de ser um fardo. Diversos trabalhos (e.g., Chu, Rogers, \& Joiner, 2016; Van Orden et al., 2012) demonstraram que os sentimentos de não pertença se relacionam com a ideação suicida através da perceção de ser um fardo.

No terceiro estudo, uma vez mais com uma amostra de estudantes universitários, a validade discriminante será testada correlacionando-se os resultados obtidos nas escalas do $I N Q-15$ com os resultados numa medida de suporte social. Esperase que os sentimentos de não pertença e a perceção de ser um fardo se correlacionem negativa e significativamente com diversas dimensões do suporte social percebido (e.g., Bell et al., 2018; Van Orden et al., 2012). De acordo com a Teoria Psicológica Interpessoal do Suicídio, os sentimentos de não pertença e a perceção de ser um fardo devem ser entendidos à luz do isolamento social (Joiner, 2005). No caso dos sentimentos de não pertença, estes relacionam-se com o isolamento social já que podem ser definidos como baixos níveis de apoio social percebido por parte dos outros (Hill \& Pettit, 2013; Van Orden et al., 2010), como um fraco sentimento de conexão social e como isolamento ou solidão (Guidry \& Cukrowicz,
2016). Relativamente à perceção de ser um fardo, está associada a um sentimento de perda de autonomia ou aumento da dependência do outro e, igualmente, ao isolamento social (Conwell \& Thompson, 2008; Van Orden \& Conwell, 2011). Assim, optou-se por estudar a relação das escalas com o suporte social (e.g., Qin \& Nordentoft, 2005; Sourander et al., 2009; Turvey et al., 2002). Será ainda realizada, neste estudo III, uma Análise Fatorial Confirmatória, com o objetivo de corroborar a estrutura fatorial obtida no estudo I.

Finalmente, no estudo quatro, será testada a estabilidade temporal do INQ-15 com um intervalo de tempo de 2 semanas. Espera-se uma correlação elevada e significativa entre os resultados obtidos nos dois momentos para as duas escalas do INQ-15.

\section{Método}

\section{Estudo I}

\section{Participantes}

Participou neste estudo uma amostra comunitária de conveniência residente em vários distritos de Portugal continental, maioritariamente do distrito de Évora (51.6\%), que participou igualmente noutros estudos publicados previamente (Campos \& Holden, 2016; Campos $\&$ Holden, 2015). Foram inicialmente contactados 484 sujeitos, tendo 58 recusado participar na investigação. Vinte e três protocolos foram considerados inválidos por falta de informação sociodemográfica ou excessivo número de missing values. Um total de 403 indivíduos com idades compreendidas entre os 18 e 67 anos $(M=37.26 ; D P=12.76)$ participaram no estudo. Duzentos e um sujeitos (49.9\%) eram do sexo feminino e $202(50.1 \%)$ eram do sexo masculino, 321 participantes $(79.7 \%)$ estavam empregados, sendo o nível médio de anos de escolaridade de $11.87(D P=3.08)$.

\section{Instrumentos}

Interpersonal Needs Questionnaire (INQ-15; Van Orden et al., 2012). É um inventário composto por 15 itens que permite avaliar os dois principais construtos da Teoria Psicológica Interpessoal do Suicídio de Joiner (2005): os 
sentimentos de não pertença e a perceção de ser um fardo. A escala de resposta é de tipo Likert com 7 pontos, em que 1 corresponde a "De maneira nenhuma verdadeiro para mim" e 7 a "Muito verdadeiro para mim". A escala de Perceção de ser um fardo é composta por 6 itens (e.g., "Atualmente, acho que sou um fardo para a sociedade"). O resultado obtém-se somando as pontuações em cada um dos seis itens e dividido esse total pelo número de itens. A escala de Sentimentos de não pertença é composta por 9 itens (e.g., "Atualmente, sinto-me desligado das outras pessoas"), sendo que seis deles são cotados de forma inversa (e.g., "Atualmente, sinto que pertenço a algo ou alguém"). O total da escala obtém-se da mesma forma que para a escala de Perceção de ser um fardo, somando os resultados nos nove itens e dividindo essa soma pelo número de itens. $O$ valor de alfa de Cronbach da versão original (Van Orden et al., 2008) foi de .80 para a escala de Sentimentos de não pertença e de .88 para a escala de Perceção de ser um fardo.

A versão original foi traduzida previamente para português por três psicólogos clínicos, incluindo o segundo autor deste trabalho. Os três psicólogos discutiram algumas dúvidas em torno da tradução de alguns itens, mas ainda assim não se notaram problemas de maior na tradução dos 15 itens. De seguida uma tradutora bilingue realizou uma retroversão para inglês. A versão original e a retroversão mostraram-se bastante semelhantes e, os quatro autores, concordaram que não seriam necessárias alterações nos itens.

Center for Epidemiologic Studies Depression Scale (CES-D; Radloff, 1977). Esta escala pretende avaliar a frequência de sintomas depressivos na semana precedente à avaliação, através de um conjunto de 20 itens (e.g., "Fiquei aborrecido com coisas que habitualmente não me aborrecem"), respondidos numa escala de Likert de 4 pontos, correspondendo 0 a "Nunca ou muito raramente" e 3 a "Com muita frequência ou sempre". Os resultados totais podem variar entre 0 e 60, com resultado mais elevados a descreverem mais sintomas depressivos. Saliente-se que existem quatro itens que são cotados de forma inversa (e.g., "Senti que valia tanto como os outros"). Na versão original (Radloff, 1977), os valores de alfa de Cronbach variaram entre $.85 \mathrm{e}$ $.90 \mathrm{em}$ várias amostras. A escala foi adaptada para a população portuguesa por Gonçalves e Fagulha (2004) e os valores de alfa variaram entre $.87 \mathrm{e}$ .92 para várias amostras. No presente estudo o valor do alfa de Cronbach foi de .91 .

Psychache Scale (Holden, Metha, Cunningham, \& McLeod, 2001). É uma escala que operacionaliza o construto de dor psicológica, psychache, tendo por base a definição de Shneidman (1996): uma dor crónica intensa, que flutua livremente e não é específica de uma situação (Mills, Green, \& Reddon, 2005). A escala é composta por 13 itens (e.g.,"A minha alma dói", "Não consigo aguentar mais a minha dor"), respondidos numa escala de Likert de 5 pontos (Campos \& Holden, 2015). Para os primeiros 9 itens, a escala de resposta refere-se a frequência, em que 1 significa "Nunca" e 5 "Sempre". Para os restantes quatro itens, a escala de resposta refere-se à concordância, variando entre 1-"Discordo fortemente" e 5-“Concordo fortemente". Os resultados neste instrumento podem variar entre 13 e 65 , traduzindo os resultados mais altos, níveis mais elevados de dor psicológica. A versão original apresenta valores de alfa de Cronbach superiores a .90 em diversas amostras (e.g., Holden et al., 2001; Troister \& Holden, 2012). A adaptação para a população portuguesa foi realizada por Campos, Holden e Gomes (2018). Para a adaptação portuguesa, é apresentado um valor de alfa de Cronbach de .97 e, no presente estudo, o valor de alfa de Cronbach é de .96.

Suicidal Behaviors Questionnaire (SBQ-R; Osman, Gutierrez, Kopper, Barrios, \& Chiros, 2001). O instrumento é composto por 4 itens para avaliar o risco suicidário, tendo por base a ideação e os comportamentos suicidas ao longo da vida. Este instrumento permite obter um valor global que pode variar entre 3 e 18, com resultados mais elevados a descreverem maior risco suicidário. Os autores da versão original reportam um ponto de corte de 7 (Osman et al., 2001) e valores de alfa de Cronbach que variam entre .76 e .88 em várias amostras. Para a versão portuguesa, da autoria de Campos e Holden (2016), é apresentado um valor de alfa de .74 e um ponto de corte, igualmente de 7. Neste estudo o alfa obtido foi de .79 . 


\section{Procedimentos}

O protocolo de investigação que foi aplicado era composto por um questionário sociodemográfico e um conjunto de outras medidas, entre as quais: o Interpersonal Needs Questionnaire; a Psychache Scale; a Center for Epidemiologic Studies Depression Scale e o Suicidal Behaviors Questionnaire Revised. Assistentes de investigação, previamente preparadas, contactaram os participantes em espaços públicos. Os participantes assinaram um termo de consentimento informado e foi-lhes dada a possibilidade de desistir a qualquer momento da participação. As instruções de participação foram transmitidas por escrito. Os instrumentos foram apresentados numa ordem variável, mas o questionário sociodemográfico foi sempre $\mathrm{o}$ primeiro a ser apresentado. A recolha de dados seguiu as recomendações deontológicas das guidelines da American Psychological Association e da Ordem dos Psicólogos Portugueses.

\section{Análise de dados}

Com recurso ao software de análise estatística IBM SPSS Statistics (versão 23) calculou-se o alfa de Cronbach para as duas escalas do $I N Q-15$, no sentido de testar a sua consistência interna. Para além disso, realizou-se uma Análise em Componentes Principais. Como heurísticas para determinar o número de fatores, recorre-se à análise paralela de Horn (1965) e a Velicer's (1976) minimum average partial correlation, utilizando-se dois softwares, o de Watkins (2006)

e de O'Connor (2000), respetivamente (Timmerman \& Lorenzo-Sava, 2011). Estas formas de determinar o número de fatores superam a regra do eigenvalue superior a 1 (Velicer, Eaton, \& Fava, 2000). Procedeu-se a uma rotação oblíqua de tipo promax aos fatores obtidos, da mesma forma que para a forma original. Calculou-se ainda a correlação entre os resultados nas escalas do $I N Q-15$ e os resultados na $C E S-D$, na Psychache Scale e no Suicidal Behaviors Questionnaire-Revised, no sentido de testar a validade convergente. Por último, avaliouse a validade referenciada pelo critério risco suicidário, comparando-se através de testes $t$ de Student os indivíduos que obtiveram um valor igual ou superior ao ponto de corte no SBQ-R (7 pontos) e os que obtiveram um resultado inferior a esse ponto de corte.

\section{Estudo II}

\section{Participantes}

Neste estudo participou uma amostra de conveniência de estudantes universitários que foi igualmente utilizada num outro estudo previamente publicado (Campos et al., 2016). A amostra inicial era composta por 440 estudantes universitários. Quarenta e quatro protocolos foram excluídos. Destes 44 protocolos, 24 pertenciam a participantes que não forneceram a identificação (as iniciais do nome de modo a emparelhar os protocolos com um segundo momento de recolha de dados, cinco meses depois) e 20 sujeitos deram um excessivo número de missing values. Assim, no primeiro momento de recolha de dados, a amostra final ficou composta por 396 indivíduos. No segundo momento, 82 sujeitos não participaram, 32 protocolos foram excluídos por impossibilidade de emparelhamento e dois protocolos foram excluídos devido a um excessivo número de missing values. A amostra final no segundo momento ficou composta por 280 participantes. A maior parte dos participantes eram mulheres (70\%), apenas $5.4 \%$ eram trabalhadores-estudantes, $\quad 72.9 \%$ estavam deslocados da residência habitual, $52.1 \%$ frequentavam o $1^{\circ}$ ano do curso e a média de idades foi de 19.73 anos $(D P=2.17)$.

\section{Instrumentos}

Interpersonal Needs Questionnaire (INQ-15; Van Orden, Cukrowicz, Witte, \& Joiner, 2012). Já descrito anteriormente.

Suicide Ideation Questionnaire (SIQ; Reynolds, 1988). O questionário é composto por 30 itens (e.g., "Pensei que seria melhor não estar vivo", "Pensei que se tivesse oportunidade me suicidaria") que avaliam a ideação suicida no mês anterior à avaliação. A escala de resposta é no formato Likert, de 7 pontos, em que 0 corresponde a "Nunca tive este pensamento" e 6 "Quase todos os dias". Os resultados variam entre 0 e 180, sendo que pontuações mais elevadas correspondem a uma ideação suicida mais grave. 
Segundo Reynolds (1988), pode considerar-se um potencial risco de suicídio se o sujeito obtiver pelo menos 41 pontos. O alfa de Cronbach da versão original é de .97. A prova foi adaptada para a população portuguesa por Ferreira e Castela (1999). O valor de alfa de Cronbach da versão portuguesa foi de $.96 \mathrm{e}$, neste estudo foi de .96 no primeiro momento e .97 no segundo momento.

\section{Procedimentos}

Os participantes voluntariaram-se para participar e preencheram um protocolo de investigação após assinarem um termo de consentimento informado. Cada protocolo era composto por vários questionários, apresentados em ordem variável, incluindo o Suicide Ideation Questionnaire e o Interpersonal Needs Questionnaire 15. As condições de participação na investigação, descritas no termo de consentimento informado, salientavam o caráter voluntário, anónimo e não remunerado da participação, para além de todos os participantes terem a possibilidade de interromper a sua participação a qualquer momento. Todos os protocolos foram recolhidos em grupo, em contexto de sala de aula, e as instruções apresentaram-se em formato escrito. A recolha de dados seguiu as recomendações deontológicas das guidelines da American Psychological Association e da Ordem dos Psicólogos Portugueses.

\section{Análise de dados}

Com recurso ao software de análise estatística IBM SPSS Statistics (versão 23) foi calculado o alfa de Cronbach para avaliar a consistência interna das escalas do INQ-15. Para além desta análise, utilizou-se o software de análise estatística IBM AMOS (versão 21) para testar a validade preditiva das escalas do INQ-15. Mais especificamente, testou-se um modelo longitudinal de path analysis (Figura 1) para prever variações na ideação suicida a cinco meses, em que se considerou a variável sentimentos de não pertença como exógena e a variável perceção de ser um fardo como mediadora. Ainda neste estudo, testou-se a validade referenciada pelo critério ideação suicida. Assim, compararam-se os resultados nas escalas do INQ-15 obtidos pelos indivíduos que obtiveram um resultado igual ou superior ao ponto de corte no SIQ - 41 pontos -, com os resultados nas escalas do INQ-15 dos indivíduos que obtiveram um resultado inferior a esse ponto de corte.

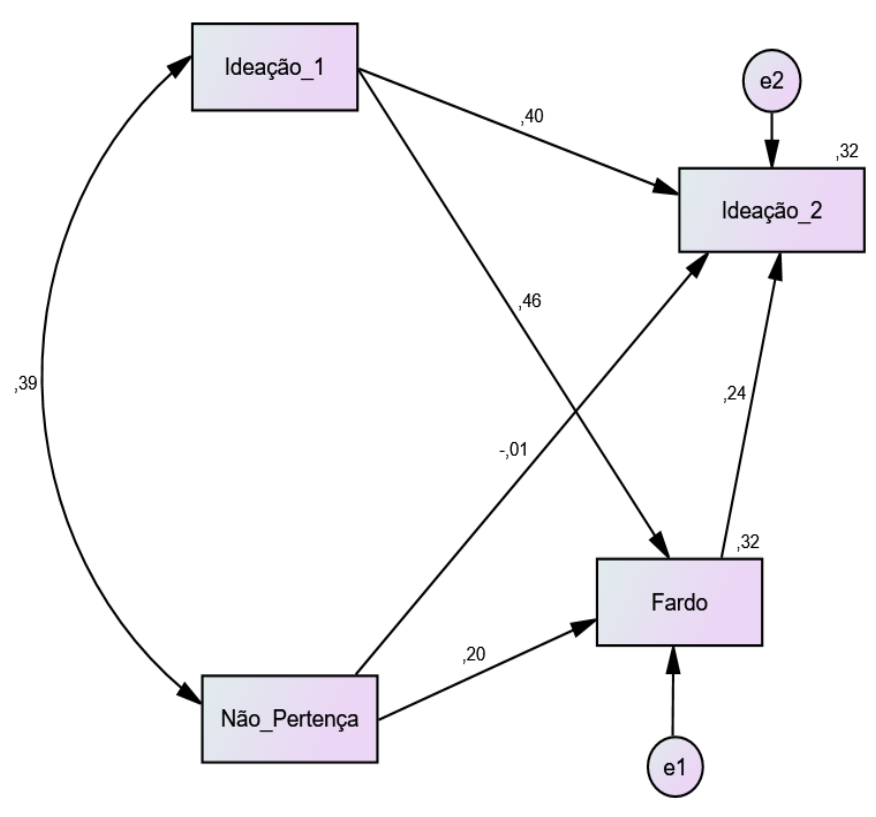

Figura 1. Modelo de path analysis testado

\section{Estudo III}

\section{Participantes}

Neste terceiro estudo participou uma amostra de conveniência de estudantes universitários. Foram contactados 349 estudantes da Universidade de Évora, com idades compreendidas entre os 18 e os 25 anos. Dos 349 estudantes, um não quis participar, seis desistiram durante o preenchimento do protocolo e 11 protocolos foram posteriormente considerados inválidos, devido a um número excessivo de missing values ou um estilo de resposta inadequado. A amostra final ficou constituída por 331 participantes, com uma média de idades de 19.89 anos $(D P=1.74)$, com maior representação do sexo feminino (56.2\%).

\section{Instrumentos}

Interpersonal Needs Questionnaire (INQ-15; Van Orden et al., 2012). Já descrito anteriormente.

Multidimensional Scale of Perceived Social Support (MSPSS; Zimet, Dahlem, Zimet, \& Farley, 1988). A escala permite uma avaliação do suporte social percebido em três áreas: família, amigos e outros significativos. Pode também ser calculado um valor global referente ao suporte 
social. Trata-se de um questionário de autorresposta composto por 12 itens, a serem respondidos numa escala de Likert de 7 pontos. Nesta escala de resposta, 1 ponto corresponde a "Discordo completamente" e 7 a "Concordo completamente". Os resultados elevados correspondem à perceção de um suporte social elevado e coeso. Exemplo de um item para a escala Família é: "A minha família tenta ajudarme verdadeiramente", para a escala Amigos: "Posso contar com os meus amigos quando algo corre mal", e para Outros Significativos: "Há uma pessoa especial na minha vida que se preocupa com os meus sentimentos". Relativamente à consistência interna, os valores de alfa de Cronbach na versão original (Zimet et al., 1988) foram os seguintes: .87 para a escala Família, .85 para a escala Amigos e .91 para a escala Outros Significativos. A adaptação para a população portuguesa foi resultado da investigação realizada por Carvalho, Pinto-Gouveia, Pimentel, Maia e Mota-Pereira (2011), tendo os autores obtido alfas de Cronbach entre de .87 e .93 para a escala Família, entre .91 e .94 para escala Amigos e entre .89 e .92 para a escala Outros Significativos, em diversas amostras. Neste estudo o alfa de Cronbach foi de .95 para Família, .94 para Amigos e .92 para Outros Significativos.

\section{Procedimentos}

Os participantes voluntariaram-se a participar na investigação e assinaram um termo de consentimento informado antes de preencherem individualmente um protocolo de investigação, composto por vários questionários, entre os quais o Interpersonal Needs Questionnaire 15 e a Multidimensional Scale of Perceived Social Support. A recolha de dados obedeceu a procedimentos idênticos aos do estudo II.

\section{Análise de dados}

Para estudar a validade discriminante do $I N Q$ foi calculada a correlação das duas escalas do INQ-15 com as três escalas do Multidimensional Scale of Perceived Social Support (MSPSS). Para corroborar a estrutura fatorial obtida no estudo I, e recorrendo ao software AMOS 21, realizou-se uma Análise Fatorial Confirmatória, através da modelação de equações estruturais. Para avaliar o ajustamento do modelo aos dados foram utilizados os seguintes índices de ajustamento: o ChiQuadrado / graus de liberdade $\left(\chi^{2} / \mathrm{df}\right)$, o CFI (Comparative Fit Index), o NFI (Normed Fit Index), o RMSEA (Root Mean Square Error of Approximation) e o SRMR (Standardized Root Mean Square Residual). Um bom ajustamento do modelo aos dados é indicado por um RMSEA inferior a.08, um SRMR igualmente inferior a .08 (Hu \& Bentler, 1999), um CFI e um NFI de pelo menos .90 (Brown, 2006) e um $\chi^{2} / \mathrm{df}$ inferior a 5 (Marôco, 2010). Utilizou-se ainda o índice AIC para comparar um modelo de dois fatores com um modelo de três fatores.

\section{Estudo IV}

\section{Participantes}

Participou neste estudo uma amostra de conveniência de estudantes universitários. No primeiro momento foram contactados 95 sujeitos. Todos os contactados aceitaram participar no estudo. No segundo momento, 2 semanas depois, apenas 77 sujeitos participaram, sendo 77 (87\%) do sexo feminino. As idades variaram entre os 19 e os $24 \operatorname{anos}(M=20.55, D P=1.30)$.

\section{Instrumentos}

Interpersonal Needs Questionnaire (INQ-15; Van Orden et al., 2012). Já descrito anteriormente.

\section{Procedimento}

Os participantes preencheram o INQ-15 em ambos os momentos, em contexto de sala de aula. A aplicação foi voluntária.

\section{Análise dos dados}

Para avaliar a estabilidade temporal do $I N Q$ 15 com o intervalo de 2 semanas, calculou-se a correlação entre os resultados em cada uma das duas escalas do instrumento nos dois momentos de recolha de dados.

\section{Resultados}

\section{Estudo I}

Os alfas de Cronbach para a amostra total de 403 participantes foram de .92 para a escala de Perceção de ser um fardo e de .80 para a escala de Sentimentos de não pertença. A simulação de Monte Carlo com 1.000 replicações indica uma solução 
Quadro 1. Matriz de estrutura para a Análise em Componentes Principais com rotação Promax aos 15 itens

\begin{tabular}{|c|c|c|c|c|}
\hline \multirow{2}{*}{ Itens } & & \multicolumn{3}{|c|}{ Componente } \\
\hline & & 1 & 2 & 3 \\
\hline 1 & $\begin{array}{l}\text { Actualmente, as pessoas que fazem parte da minha vida } \\
\text { estariam melhores se eu desaparecesse }\end{array}$ & .902 & -.281 & .468 \\
\hline 2 & $\begin{array}{l}\text { Actualmente, as pessoas que fazem parte da minha vida } \\
\text { estariam mais felizes sem mim }\end{array}$ & .883 & -.278 & .481 \\
\hline 3 & Actualmente, acho que sou um fardo para a sociedade & .770 & -.268 & .438 \\
\hline 4 & $\begin{array}{l}\text { Actualmente, acho que a minha morte seria um alívio para as } \\
\text { pessoas que fazem parte da minha vida }\end{array}$ & .804 & -.321 & .426 \\
\hline 5 & $\begin{array}{l}\text { Actualmente, acho que as pessoas que fazem parte da minha } \\
\text { vida desejariam ver-se livres de mim }\end{array}$ & .800 & -.233 & .390 \\
\hline 6 & $\begin{array}{l}\text { Actualmente, acho que pioro as coisas para as pessoas que } \\
\text { fazem parte da minha vida }\end{array}$ & .869 & -.227 & .516 \\
\hline 7 & Actualmente, as outras pessoas preocupam-se comigo & -.179 & 689 & -.002 \\
\hline 8 & Actualmente, sinto que pertenço a algo ou alguém & -.159 & .678 & .032 \\
\hline 9 & $\begin{array}{l}\text { Actualmente, raramente interajo com as pessoas que se } \\
\text { preocupam comigo }\end{array}$ & .341 & -.129 & .649 \\
\hline 10 & $\begin{array}{l}\text { Actualmente, tenho sorte em ter muitos amigos que cuidam de } \\
\text { mim e me dão apoio }\end{array}$ & -.239 & .708 & -.351 \\
\hline 11 & Actualmente, sinto-me desligado das outras pessoas & .449 & -.333 & .811 \\
\hline 12 & $\begin{array}{l}\text { Actualmente, sinto-me com frequência como um estranho } \\
\text { quando estou no meio de outras pessoas }\end{array}$ & .513 & -.221 & .797 \\
\hline 13 & $\begin{array}{l}\text { Actualmente, sinto que há pessoas a quem posso recorrer em } \\
\text { caso de necessidade }\end{array}$ & -.202 & .796 & -.379 \\
\hline 14 & Actualmente, estou próximo das outras pessoas & -.374 & .772 & -.640 \\
\hline 15 & $\begin{array}{l}\text { Actualmente, tenho pelo menos uma interacção satisfatória } \\
\text { com alguém, todos os dias }\end{array}$ & -.270 & .659 & -.509 \\
\hline
\end{tabular}

Nota. Os valores assinalados a negrito representam a saturação mais elevada do item no respetivo fator.

fatorial de três fatores, bem como a heurística minimum average partial correlation de Velicer, bem como o próprio critério de Kaiser. Os quatro primeiros valores próprios para os dados foram: $5.991,2.338,1.227,0.866$. Na simulação de Monte Carlo, os quatro primeiros valores próprios foram: $1.341,1.265,1.206,1.156$. Os três primeiros fatores explicam um total de $63.71 \%$ da variância. Após rotação oblíqua promax todos os itens apresentam saturações iguais ou superiores a $.65 \mathrm{em}$ apenas um dos fatores. A matriz de estrutura encontra-se no quadro 1 . Os valores de alfa de Cronbach para os fatores 1, 2 e 3 foram, respetivamente de $.92, .81$ e .72 .

A escala de Perceção de ser um fardo correlacionou-se significativamente com a CESD, $r=.49$, com a Psychache Scale, $r=.46$ e com o SBQ-R $r=.50$. A escala de Sentimentos de não pertença correlacionou-se significativamente com a CES-D, $r=.43$ com a Psychache Scale, $r=.36 \mathrm{e}$ com o SBQ-R $r=.35$.

Para a amostra total o valor médio na escala de Perceção de ser um fardo foi de 1.41 ( $D P=.80)$ e na escala de Sentimentos de não pertença foi de $2.60 \quad(D P=1.03)$. Ao comparar o grupo de indivíduos com resultados iguais ou superiores ao ponto de corte no SBQ-R $(n=89)$ com os indivíduos com resultados abaixo do ponto de corte $(n=314)$ relativamente ao resultado na escala de Perceção de ser um fardo, verifica-se que os primeiros apresentam resultados significativamente superiores $(M=1.97, D P=1.22)$ relativamente aos segundos $(M=1.25, D P=.54)$, $t_{(401)}=5.42, p<.001, \mathrm{~d}$ de Cohen $=0.97$. Por sua vez, ao comparar o grupo de indivíduos com resultados iguais ou superiores ao ponto de corte no SBQ-R com os indivíduos com resultados abaixo do ponto de corte relativamente ao resultado na escala de Sentimentos de não pertença, verifica-se que os primeiros apresentam resultados significativamente superiores $(M=3.20$, $D P=1.09)$ em relação aos segundos $(M=2.44$, $D P=.95), t_{(401)}=6.51, p<.001, \mathrm{~d}$ de Cohen $=.77$.

\section{Estudo II}

Os alfas de Cronbach para a amostra final de 280 participantes foram de .91 para a escala de Perceção de ser um fardo e de .83 para a escala de Sentimentos de não pertença. Quando se considera os seis itens de cotação inversa desta escala, na verdade os que saturam no fator II, o 
alfa é de .86 e, quando se considera apenas os três itens de cotação direta, fator III, o alfa é de .71.

No modelo de path analysis representado na Figura 1 verifica-se um efeito indireto dos sentimentos de não pertença na ideação suicida avaliada no momento 2 através da perceção de ser um fardo $(\beta=.05, t=2.38, p<.01, \mathrm{EP}=.021 ; 95 \% \mathrm{CI}$ $[0.014,0.010], p<.01)$, um efeito direto dos sentimentos de não pertença na perceção de ser um fardo $(\beta=.20, t=3.17, p<.001, \mathrm{EP}=.063 ; 95 \%$ CI [0.082, 0.329], $p<.001)$, e um efeito direto da perceção de ser um fardo na ideação suicida no momento $2(\beta=.24, t=2.72, p<.01, \mathrm{EP}=.088 ; 95 \%$ CI [0.064, 0.404], $p<.05)$. Verifica-se igualmente um efeito direto da ideação suicida avaliada no momento 1 na ideação suicida avaliada no momento $2(\beta=.40, t=4.55, p<.001, \mathrm{EP}=.088: 95 \%$ CI [0.252, 0.588], $p<.001)$, mas não um efeito direto dos sentimentos de não pertença na ideação no momento 2 .

Para a amostra final, o valor médio na escala de Perceção de ser um fardo foi de $1.26(D P=.58)$ e na escala de Sentimentos de não pertença foi de $2.35(D P=1.03)$. Ao comparar o grupo de indivíduos com resultados iguais ou superiores ao ponto de corte no SIQ obtido no momento 2 $(n=18)$ com os indivíduos com resultados abaixo do ponto de corte $(n=262)$ relativamente ao resultado na escala de Perceção de ser um fardo, verifica-se que os primeiros apresentam resultados significativamente superiores $(M=2.19, D P=.94)$, relativamente aos segundos $(M=1.20, D P=.48)$, $t_{(278)}=4.47, p<.001, \mathrm{~d}$ de Cohen $=1.60$. Por sua vez, ao comparar o grupo de indivíduos com resultados iguais ou superiores ao ponto de corte no SIQ com os indivíduos com resultados abaixo do ponto de corte relativamente ao resultado na escala de Sentimentos de não pertença, verifica-se que os primeiros apresentam resultados significativamente superiores $(M=3.10, D P=1.24)$ aos segundos $(M=2.30, D P=1.00), t_{(278)}=3.18$, $p<.01, \mathrm{~d}$ de Cohen $=.75$.

\section{Estudo III}

O alfa de Cronbach para a amostra total de 331 participantes foi de .93 para a escala de Perceção de ser um fardo e de .85 para a escala de Sentimentos de não pertença. Quando se considera os seis itens de cotação inversa, os que saturam no fator II, o alfa é de .86, e quando se considera apenas os três itens de cotação direta, fator III, o alfa é de .71 .

A escala de Perceção de ser um fardo correlacionou-se negativa e significativamente com a escala de Suporte social percebido Família, $r=-.47$, com a escala de Suporte social percebido - Amigos $r=-.43$ e com escala de Suporte social percebido - Outros significativos $r=-.38$. A escala de Sentimentos de não pertença correlacionou-se negativa e significativamente com a escala de Suporte social percebido Família, $r=-.40$, com a escala de Suporte social percebido - Amigos $r=-.68$, escala de Suporte social percebido - Outros significativos $r=-.53$.

No sentido de corroborar a estrutura de três fatores obtida no estudo I, realizou-se uma Análise Fatorial Confirmatória. O ajustamento do modelo aos dados é aceitável $\left(\chi^{2} / \mathrm{df}=3.422\right.$, $\mathrm{CFI}=.934, \mathrm{NFI}=.910 ; \mathrm{RMSEA}=.086, \mathrm{SRMR}=.059$; $\mathrm{AIC}=363.71)$. O modelo está representado na Figura 2. As correlações entre os fatores são significativas. Quando se testou um modelo de dois fatores, com o fator I incluindo os seis itens da escala de Perceção de ser um fardo e fator II incluindo os nove itens da escala de Sentimentos de não pertença, o modelo ajustou-se mal aos dados $\quad\left(\chi^{2} / \mathrm{df}=5.4642, \quad\right.$ CFI $=.871, \quad \mathrm{NFI}=.848 ;$ RMSEA=.119, $\mathrm{SRMR=.104;} \mathrm{AIC}=564.14)$.

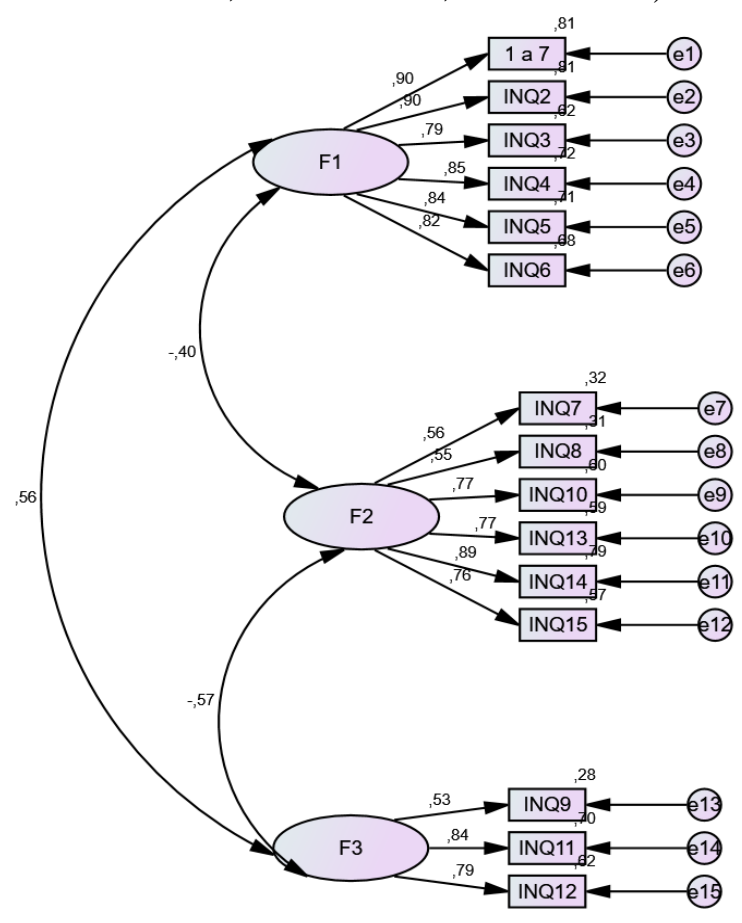

Figura 2. Representação gráfica do modelo de análise fatorial confirmatória 


\section{Estudo IV}

A correlação entre os resultados obtidos na escala de Perceção de ser um fardo nos dois momentos, com um intervalo de tempo de 2 semanas, foi de $r=.78$. A correlação para a escala de Sentimentos de não pertença foi de $r=.92$.

\section{Discussão}

Este trabalho teve como objetivo apresentar estudos de validação da versão portuguesa do Interpersonal Needs Questionnaire 15, com diferentes amostras. Testou-se a estrutura fatorial da prova, através de Análise em Componentes Principais, de cariz exploratório e, da Análise Fatorial Confirmatória, a consistência interna, a validade convergente, preditiva e referenciada pelos critérios risco suicidário e ideação suicida, a validade discriminante e a estabilidade temporal com um intervalo de 2 semanas.

Obtiveram-se valores de consistência interna elevados para ambas as escalas do inventário, de forma similar ao que acontece com a versão original (Van Orden et al., 2012) onde os alfas de Cronbach foram de .85 para a escala de Sentimentos de não pertença e .89 para a escala de Perceção de ser um fardo. Com as amostras dos estudos I, II e III e, para a escala de Sentimentos de não pertença, os valores de alfa variaram entre .80 e .88 e, para a escala de Perceção de ser um fardo, variaram entre .91 e .93. Os valores da forma original e da forma portuguesa são comparáveis, já que, como acontece na versão original, os valores de alfa de Cronbach na versão portuguesa são ligeiramente inferiores para escala de Sentimentos de não pertença comparativamente à escala de Perceção de ser um fardo.

Relativamente à estabilidade temporal, no estudo IV obteve-se uma correlação mais elevada para a escala de Sentimentos de não pertença do que para a escala de Perceção de ser um fardo. Embora ambos os construtos constituam estados cognitivo-afetivos dinâmicos, espera-se que a perceção de ser um fardo apresente uma ainda maior variabilidade, já que sofre mais influência dos relacionamentos do indivíduo (Van Orden et al., 2010).

No que se refere à estrutura fatorial, obtiveram-se 3 fatores no estudo I, estrutura que foi confirmada no estudo III. De acordo com a hipótese formulada, seria de esperar encontrar dois fatores, cada um representando um dos dois construtos operacionalizados no INQ-15 (perceção de ser um fardo e sentimentos de não pertença), tal como se verificou nos estudos realizados com a versão original (Van Orden et al., 2012). Na versão portuguesa, no primeiro fator saturam os seis itens que foram redigidos para avaliar a perceção de ser um fardo; já os restantes nove itens, redigidos para operacionalizar os sentimentos de não pertença, saturam em dois fatores distintos: os itens 7, 8, 10, 13, 14 e 15 escritos no sentido "da pertença" e, portanto, cotados de forma inversa, saturam no segundo fator e, os itens 9,11 e 12 , cotados de forma direta, saturam num terceiro fator. A Análise Fatorial Confirmatória corroborou uma estrutura de três fatores e o ajustamento do modelo de três fatores aos dados foi satisfatório. Por outro lado, quando se testou um modelo de dois fatores, o ajustamento do modelo aos dados mostrou-se insuficiente.

A "divisão" em dois fatores dos itens que avaliam os sentimentos de não pertença poderá estar associada ao formato dos itens: uma parte dos itens cota no sentido inverso. No entanto, uma magnitude moderada da correlação entre o fator II e o fator III (0.57), sendo idêntica à do fator III com o fator I (0.56) faz pensar que o formato dos itens poderá não ser uma justificação suficiente para explicar a estrutura de três fatores. Pode assim colocar-se a hipótese dos itens dos fatores II e III avaliarem, de facto, dois constructos diferentes. Com isto quer dizer-se que, os itens que saturam no fator II (e.g., "Actualmente, as outras pessoas preocupam-se comigo") poderão não medir "apenas" o inverso dos itens do fator III (e.g. "Actualmente, raramente interajo com as pessoas que se preocupam comigo"), ou seja o inverso dos sentimentos de não pertença como seria de esperar, mas uma outra dimensão, possivelmente mais relacionada com o suporte social. Esta diferença entre as estruturas fatoriais da versão portuguesa e da forma original pode refletir diferenças culturais a explorar em estudos futuros. Mas pode simplesmente ter acontecido também, como aliás com medidas de outros construtos psicológicos, que os itens quando apresentados num "sentido inverso" acabem por 
saturar num fator diferente daqueles que aparecem escritos num "sentido direto".

Relativamente à validade referenciada por um critério, e tal como esperado, verificou-se no estudo I que os indivíduos que obtiveram 7 ou mais pontos na medida de risco suicidário, obtiveram resultados significativamente superiores nas duas escalas do INQ-15. Este resultado evidência que estes dois construtos (perceção de ser um fardo e sentimentos de não pertença) poderão constituir fatores que contribuem para o risco suicidário (Anestis, Khazem, Mohn, \& Green, 2015; Van Orden et al., 2012). De acordo com a teoria de Joiner (2005), a ideação e o desejo suicidário são distintos das tentativas (sejam estas letais ou não letais) (Van Orden et al., 2010). No entanto, as duas variáveis interpessoais operacionalizadas pelo INQ-15 poderão ser correlatos importantes da motivação suicida. Testou-se também a validade referenciada pelo critério de ideação suicida no estudo II. Verificou-se, tal como esperado, que os indivíduos que obtiveram 41 ou mais pontos na medida de ideação suicida, o SIQ, pontuaram significativamente mais alto nas escalas do INQ15. Este resultado corrobora o anterior e, também, a hipótese de que a perceção de ser um fardo e os sentimentos de não pertença são correlatos da ideação suicida (Joiner, 2005; Van Orden et al., 2010).

Testou-se também no estudo II um modelo longitudinal de path analysis, para avaliar a validade preditiva das escalas do INQ-15. Nesta análise verificou-se uma relação direta entre a perceção de ser um fardo e variações na ideação suicida e, uma relação indireta entre sentimentos de não pertença e variações na ideação suicida ao longo de 5 meses. Outros estudos já tinham demonstrado o efeito direto da perceção de ser um fardo na ideação suicida (e.g., Brown, Dahlen, Mills, Rick, \& Biblarz ,1999; Garza \& Petit, 2010; Jahn \& Cukrowicz, 2011) e o efeito indireto dos sentimentos de não pertença na ideação suicida (e.g., Campos et al., 2016; Hill \& Petit, 2013) e, de que os sentimentos de não pertença só se relacionam com a ideação suicida através da perceção de ser um fardo (e.g., Cero, Zuromski, Witte, Ribeiro, \& Joiner, 2015; Lamis, Leenaars, Jahn, \& Lester, 2013).

Já no que diz respeito à relação dos resultados nas escalas do INQ com os resultados noutras provas psicológicas, a relação positiva entre os resultados nas duas escalas do INQ-15 e a sintomatologia depressiva no estudo I era expectável, se tivermos em conta resultados previamente obtidos (e.g., Campos et al., 2016; Kleiman, Riskind, Stange, Hamilton, \& Alloy, 2014; Campos \& Holden, 2016; Mitchell et al., 2018). A teoria interpessoal prevê uma relação significativa dos construtos avaliados pelo INQ e a psicopatologia (Joiner, 2005; Joiner et al., 2009). Sobre a relação das duas escalas do INQ15 com a dor psicológica, esta pode ser explicada pela própria conceção de Joiner (2005), já que os dois construtos interpessoais são considerados como o "colapsar" em dois clusters daquilo que Shneidman (1996) descrevera como a impossibilidade de satisfação de necessidades psicológicas fundamentais, resultando em dor psicológica, em última instância, responsável pelo desejo de morrer.

Por fim, avaliou-se a validade discriminante no estudo III, correlacionando as duas escalas do INQ-15 com as três escalas do MSPSS (Família, Amigos e Outros significativos). Os resultados foram ao encontro do esperado, já que se verificou uma correlação negativa e significativa entre as duas escalas do INQ-15 e as três escalas da MSPSS. No entanto, pode questionar-se se estas correlações negativas são de facto uma evidência da validade discriminante das escalas do INQ ou, uma vez mais, da sua validade convergente. O que estas correlações mostram é que de facto há uma relação forte entre a perceção de ser um fardo e os sentimentos de não pertença com o suporte social, só que se trata de uma relação inversa. Estes resultados foram no sentido do que já se tinha verificado em estudos anteriores (e.g., Qin \& Nordentoft, 2005; Sourander et al., 2009; Turvey et al., 2002) e são suportados pela fundamentação teórica que relaciona sentimentos de não pertença e perceção de ser um fardo com um baixo sentimento de conexão social (Guidry \& Cukrowicz, 2016) e com o isolamento (Conwell \& Thompson, 2008; Van Orden \& Conwell, 2011). Note-se ainda que, as correlações entre as escalas do INQ-15 e as escalas de suporte social foram mais elevadas no caso do suporte social percebido dos amigos, o que pode ter que ver com a amostra em estudo, de jovens adultos e, justamente, com a 
importância que os amigos podem ter neste período de desenvolvimento.

\section{Conclusões, limitações e estudos futuros}

Contrariamente ao que acontece com a forma original, a análise fatorial, quer exploratória, quer confirmatória, apontam para uma estrutura de três fatores, e não de dois. Estudos futuros, nomeadamente com amostras de risco elevado, poderão ajudar a esclarecer se se trata de fatores que captam uma variância relacionada apenas com o formato dos itens, ou se algum outro aspeto poderá ser responsável por esta diferença relativamente à forma original. Mais do que simplesmente o inverso dos sentimentos de não pertença, os itens de cotação inversa que na forma portuguesa saturam no fator II poderão constituir, na verdade, uma medida de suporte social e este pode não ser apenas o contrário da não pertença. Sentir pertença e perceber suporte social poderão ser "coisas" distintas, embora relacionadas. Numa análise auxiliar correlacionamos os fatores II e III do INQ-15 com as três escalas da medida de suporte social, o MSPSS, bem como, com o seu resultado total. À exceção da escala de Família, as correlações apresentam uma magnitude sempre mais elevada com o Fator II do que com o Fator III.

Em estudos futuros seria interessante testar a validade discriminante com uma variável ou um conjunto de variáveis que conceptualmente e empiricamente não se relacionem, quer com os sentimentos de não pertença, quer com a perceção de ser um fardo. As emoções positivas poderão ser um exemplo disso.

A presente investigação tem como limitações o facto de se ter utilizado exclusivamente amostras não clínicas de conveniência. Pode ainda referir-se o atrito no estudo II. Refira-se ainda que se pode considerar não ter efetivamente testado a validade discriminante.

De qualquer forma, os resultados apontam no sentido da forma portuguesa do INQ-15 poder ser utilizada como uma medida de motivação suicida, que assumirá especial relevância quando os indivíduos estão pouco disponíveis para revelar ao clínico essa motivação, se questionados diretamente em entrevista, ou através de itens mais óbvios das escalas de ideação suicida habitualmente utilizadas. Vale dizer que a adaptação e validação de instrumentos de avaliação é sempre uma tarefa de investigação importante, especialmente se essas versões adaptadas puderem ser usadas na prática clínica e puderem também contribuir para o "retocar" de modelos teóricos existentes (Casullo, 2009), independentemente do modelo teórico subjacente à prática de avaliação que se utilize (cf. Campos, 2017).

\section{Referências}

Anestis, J. C., Anestis, M. D., Rufino, K. A., Cramer, R. J., Miller, H., Khazem, L. R., \& Joiner, T. E. (2016). Understanding the relationship between suicidality and psychopathy: An examination of the interpersonal-psychological theory of suicidal behavior. Archives of Suicide Research, 20, 349-368.

Anestis, M. D., Bagge, C. L., Tull, M. T., \& Joiner, T. E. (2011). Clarifying the role of emotion dysregulation in the InterpersonalPsychological Theory of Suicidal Behavior in an undergraduate sample. Journal of Psychiatric Research, 45, 603-611.

Anestis, M. D., \& Joiner, T. E. (2011). Examining the role of emotion in suicidality: Negative urgency as na amplifier of the relationship between components of the InterpersonalPsychological Theory of Suicidal Behavior and lifetime number of suicide attempts. Journal of Affective Disorders, 129, 261-269.

Anestis, M. D., Khazem, L. R., Mohn, R. S., \& Green, B. A. (2015). Testing the main hypotheses of the interpersonal-psychological theory of suicidal behavior in a large diverse sample of United States military personnel. Comprehensive Psychiatry, 60, 78-85.

Baams, L., Grossman, A. H., \& Russell, S. T. (2015). Minority stress and mechanisms of risk for depression and suicidal ideation among lesbian, gay, and bisexual youth. Developmental Psychology, 51, 688-696.

Bell, C. M., Ridley, J. A., Overholser, J. C., Young, K., Athey, A., Lehmann, J., \& Phillips, K. (2018). The role of perceived burden on social support in suicide and depression. Suicide and Life-Threatning Behavior, 48, 87-94. 
Brown, T. A. (2006). Confirmatory factor analysis for applied research. New York: Guilford.

Brown, R. M., Dahlen, E., Mills, C., Rick, J., \& Biblarz, A. (1999). Evaluation of an evolutionary model of self-preservation and self-destruction. Suicide and Life-Threatening Behavior, 29, 58-71.

Bryan, C. J. (2011). The clinical utility of a brief measure of perceived burdensomeness and thwarted belongingness for the detection of suicidal military personnel. Journal of Clinical Psychology, 67, 981-992.

Campos, R. C. (2017). Do processo de avaliação da personalidade em contextos clínicos ao diagnóstico psicodinâmico: Contributos para uma avaliação psicológica psicodinâmica. Revista Iberoamericana de Diagnóstico y Evaluación - e Avaliação Psicológica, 4, 4456.

Campos, R. C., \& Holden, R. R. (2015). Testing models relating rejection, depression, interpersonal needs and psychache to suicide risk in nonclinical individuals. Journal of Clinical Psychology, 71, 994-1003.

Campos, R. C., \& Holden R. R. (2016). Portuguese version of the Suicidal Behaviors Questionnaire-Revised. Validation data and the establishment of a cut-score for screening purposes. European Journal of Psychological Assessment. Advanced online publication doi.org/10.1027/1015-5759/a000385.

Campos, R. C., \& Holden, R. R. (2016b). Testing a theory based model of suicidality in a community sample. Omega: Journal of Death and Dying. 74, 119-137.

Campos, R C., Holden, R. R., Laranjeira, P., Troister, T., Oliveira, A. R., Costa, F., Abreu, M., \& Fresca, N. (2016). Self-report depressive symptoms do not directly predict suicide in nonclinical individuals: Contributions toward a more psychosocial approach to suicide risk. Death Studies, 40, 335-349.

Campos, R. C., Holden, R. R., \& Gomes, M. (2018). Assessing psychache as a suicide risk variable: Data with the Portuguese version of the Psychache Scale. Death Studies. doi:10.1080/07481187.2018.149300
Carvalho, S., Pinto-Gouveia, J., Pimentel, P., Maia, D., \& Mota-Pereira, J. (2011). Características psicométricas da versão portuguesa da Escala Multidimensional de Suporte Social Percebido (Multidimensional Scale of Perceived Social Support - MSPSS). Psychologica, 54, 309-358.

Cero, I., Zuromski, K. L., Witte, T. K., Ribeiro, J. D., \& Joiner, T. E. (2015). Perceived burdensomeness, thwarted belongingness, and suicide ideation: Re-examination of the Interpersonal-Psychological Theory in two samples. Psychiatry Research, 228, 544-550.

Chu, C., Buchman-Schmitt, J. M., Stanley, I. H., Hom, M. A., Tucker, R. P., Hagan, C. R., \& Joiner, T. E., Jr. (2017). The Interpersonal Theory of Suicide: A systematic review and meta-analysis of a decade of cross-national research. Psychological Bulletin, 143, 13131345.

Chu, C., Rogers, M. L., \& Joiner, T. E. (2016). Cross-sectional and temporal association between non-suicidal self-injury and suicidal ideation in young adults: The explanatory roles of thwarted belongingness and perceived burdensomeness. Psychiatry Research, 246, 573-580.

Cole, A., Wingate, L., Slish, M., Tucker, R., Hollingsworth, D., \& O'Keefe, V. (2013). Burdensomeness, depression, and suicide in a sample of American-Indian college students. Ethnicity and Inequalities in Health and Social Care, 6, 77-86.

Conwell, Y., \& Thompson, C. (2008). Suicidal behavior in elders. Psychiatric Clinics of North America, 31, 333-356.

Ferreira, J., \& Castela, M. (1999). Questionário de Ideação Suicida (QIS). In M. R. Simões, M. Gonçalves, \& L. Almeida (Eds.), Testes e provas psicológicas em Portugal (pp. 123130). Braga: Sistemas Humanos e Organizacionais, Lda.

Freedenthal, S., Lamis, D. A., Osman, A., Kahlo, D., \& Gutierrez, P. M. (2011). Evaluation of the psychometric properties of the Interpersonal Needs Questionnaire-12 in samples of men and women. Journal of Clinical Psychology, 67, 609-623.

Garza, M. J., \& Petit, J. W. (2010). Perceived burdensomeness, familism, and suicidal 
ideation among Mexican women: Enhancing understanding of risk and protective factors. Suicide and Life-Threatening Behavior, 40, 567-573.

Gonçalves, B., \& Fagulha, T. (2004). The Portuguese version of the Center for Epidemiologic Studies Depression Scale (CES-D). European Journal of Psychological Assessment, 20, 339-348.

Guidry, E. T., \& Cukrowicz, K. C. (2016). Death ideation in older adults: Psychological symptoms of depression, thwarted belongingness, and perceived burdensomeness. Aging \& Mental Health, 20, 823-830.

Hagerty, B. M., Lynch-Sauer, J., Patusky, K. L., Bouwsema, M., \& Collier, P. (1992). Sense of belonging: A vital mental health concept. Archives of Psychiatric Nursing, 6, 172-177.

Hill, R. M., \& Pettit, J. W. (2013). The role of autonomy needs in suicidal ideation: Integrating The Interpersonal-Psychological Theory of Suicide and self-determination theory. Archives of Suicide Research, 17, 288301.

Hill, R. M., Rey, Y., Marin, C. E., Sharp, C., Green, K. L., \& Petit, J. W. (2015). Evaluating the Interpersonal Needs Questionnaire: Comparison of the reliability, factor structure, and predictive validity across five versions. Suicide and Life-Threatning Behavior, 45, 302-314.

Holden, R. R., Mehta, K., Cunningham, E. J., \& McLeod, L. D. (2001). Development and preliminary validation of a Scale of Psychache. Canadian Journal of Behavioural Science, 33, 224-232.

Horn, J. L. (1965). A rationale and test for the number of factors in factor analysis. Psychometrika, 30, 179-185.

Hu, L., \& Bentler, P. (1999). Cutoff criteria for fit indexes in covariance structure analysis: Conventional criteria versus new alternatives. Structural Equation Modelling, 6, 1-55,

Jahn, D. R. \& Cukrowicz, K. C. (2011). The impact of the nature of relationships on perceived burdensomeness and suicide ideation in a community sample of older adults. Suicide and Life-Threatening Behavior, 41, 635-649.
Joiner, T. E. (2005). Why people die by suicide. Cambridge, MA: Harvard University Press.

Joiner, T., Conwell, Y., Fitzpatrick, K. K., Witte, T. K., Schmidt, N. B., Berlim, M. T., Fleck, M. P. A., \& Rudd, M. D. (2005). Four studies on how past and current suicidality relate even when "everything but the kitchen sink" is covaried. Journal of Abnormal Psychology, 114, 291-303.

Joiner, T. E., Van Orden, K. A., Witte, T. K., Selby, E. A., Ribeiro, J., Lewis, R., \& Rudd, M. D. (2009). Main predictions of the interpersonal-psychological theory of suicidal behavior: Empirical tests in two samples of young adults. Journal of Abnormal Psychology, 118, 634-646.

Kleiman, E. M., Riskind, J. H., Stange, J. P., Hamilton, J. L., \& Alloy, L. B. (2014). Cognitive and interpersonal vulnerability to suicidal ideation: A weakest link approach. Behavior Therapy, 45, 778-790.

Lamis, D. A., Leenaars, L. S., Jahn, D. R., \& Lester, D. (2013). Intimate partner violence: Are perpetrators also victims and are they more likely to experience suicide ideation? Journal of Interpersonal Violence, 28, 31093128.

Marôco, J. (2010). Análise de equações estruturais: Fundamentos teóricos, software e aplicações. Lisboa: Report Number.

Marty, M. A., Segal, D. L., Coolidge, F. L., \& Klebe, K. J. (2012). Analysis of the psychometric properties of the Interpersonal Needs Questionnaire (INQ) among community-dwelling older adults. Journal of Clinical Psychology, 68, 1008-1018.

Mills, J. F., Green, K., \& Reddon, J. R. (2005). An evaluation of the Psychache Scale on an offender population. Suicide and LifeThreatening Behavior, 35, 570-580.

Mitchell, S. M., Seegan, P. L., Roush, J. F., Brown, S. L., Sustaíta, M. A., \& Cukrowicz, K. C. (2018). Retrospective cyberbullying and suicide ideation the mediating roles of depressive symptoms, perceived burdensomeness, and thwarted belongingness. Journal of Interpersonal Violence, 33, 26022620

O'Connor, B. P. (2000). SPSS and SAS programs for determining the number of components 
using parallel analysis and Velicer's MAP test. Behavior Research Methods, Instrumentation, and Computers, 32, 396-402.

Osman, A., Gutierrez, P. M., Kopper, B. A., Barrios, F. X., \& Chiros, C. E. (1998). The Positive and Negative Suicide Ideation Inventory: Development and validation. Psychological Reports, 82, 783-793.

Qin, P., \& Nordentoft, M. (2005). Suicide risk in relation to psychiatric hospitalization. Archives of General Psychiatry, 62, 427-432.

Radloff, L. S. (1977). The CES-D scale: A selfreport depression scale for research in the general population. Applied Psychological Measurement, 1, 385-401.

Reynolds, W. (1988). Suicidal Ideation Questionnaire (SIQ): Professional manual. Odessa, FL: Psychological Assessment Resources.

Shneidman, E. S. (1996). The suicidal mind. New York: Oxford University Press.

Sourander, A., Klomek, A. B., Niemela, S., Haavisto, A., Gyllenberg, D., Helenius, H., \& Gould, M. S. (2009). Childhood predictors of completed and severe suicide attempts: Findings from the Finnish 1981 Birth Cohort Study. Archives of General Psychiatry, 66, 398-406.

Timmerman, M. E., \& Lorenzo-Seva, U. (2011). Dimensionality assessment of ordered polytomous items with parallel analysis. Psychological Methods, 16, 209-220.

Troister, T., \& Holden, R. R. (2012). A two-year prospective study of psychache and its relationship to suicidality among high-risk undergraduates. Journal of Clinical Psychology, 69, 1019-1027.

Turvey, C. L., Conwell, Y., Jones, M. P., Phillips, C., Simonsick, E., Pearson, J. L., \& Wallace, R. (2002). Risk factors for late-life suicide: A prospective community-based study. American Journal of Geriatric Psychiatry, 10, 398-406.

Van Orden, K. A. (2009). Construct validity of the interpersonal needs questionnaire. Dissertation, Florida.

Van Orden, K. \& Conwell, Y. (2011). Suicides in late life. Current Psychiatry Reports, 13, 234241.
Van Orden, K. A., Bamonti, P. M., King, D. A., \& Duberstein, P. R. (2012). Does perceived burdensomeness erode meaning in life among older adults? Aging \& Mental Health, 16, 855-860.

Van Orden, K. A., Cukrowicz, K. C., Witte, T. K., \& Joiner, T. E. (2012). Thwarted belongingness and perceived burdensomeness: Construct validity and psychometric properties of the Interpersonal Needs Questionnaire. Psychological Assessment, 24, 197-215.

Van Orden, K. A., Witte, T. K., Cukrowicz, K. C., Braithwaite, S. R., Shelby, E. A., \& Joiner Jr., T. E. (2010). The Interpersonal Theory of Suicide. Psychological Review, 117, 575-600.

Van Orden, K. A., Witte, T. K., James, L. M., Castro, Y., Gordon, K. H., Braithwaite, S. R., \& Joiner, T. E., Jr. (2008). Suicidal ideation in college students varies across semesters: The mediating role of belongingness. Suicide and Life-Threatening Behavior, 38, 427-435.

Velicer, W. F. (1976). Determining the number of components from the matrix of partial correlations. Psychometrika, 31, 321-327.

Velicer, W. F., Eaton, C. A., \& Fava, J. L. (2000). Construct explication through factor or components analysis: A review and evaluation of alternative procedures for determining the number of factors or components. In R. D. Goffin \& E. Helmes (Eds.), Problems and solutions in human assessment: Honoring Douglas N. Jackson at seventy (pp. 41-71). New York: Kluwer.

Watkins, M. W. (2006). Determining Parallel Analysis criteria. Journal of Modern Applied Statistical Methods, 5, 344-346.

Zimet, G. D., Dahlem, N., Zimet, S., \& Farley, G. (1988). The Multidimensional Scale of Perceived Social Support. Journal of Personality Assessment, 52, 30-41 